\title{
Artificial Neural Network Application for Thermal Image Based Condition Monitoring of Zinc Oxide Surge Arresters
}

\author{
Novizon $^{1 \star}$, Zulkurnain Abdul-Malek ${ }^{2}$, Aulia $^{3}$ \\ ${ }^{1,2}$ Departement of Electrical Engineering, Engineering Faculty, Andalas University, Padang, Indonesia \\ ${ }^{3}$ Institute of High Voltage \& High Current, Fakulty of Electrical Engineering, Universiti Teknologi Malaysia, \\ 81310 Johor Bahru, Johor, Malaysia \\ ${ }^{*}$ Corresponding author, e-mail: unand@ac.id
}

\begin{abstract}
Manual analysis of thermal image for detecting defects and classifying of condition of surge arrester take a long time. Artificial neural network is good tool for predict and classify data. This study applied neural network for classify the degree of degradation of surge arrester. Thermal image as input of neural network was segmented using Otsu's segmentation and histogram method to get features of thermal image. Leakage current as a target of supervise neural network was extracted and applied Fast Fourier Transform to get third harmonic of resistive leakage current. The classification results meet satisfaction with error about $3 \%$.
\end{abstract}

Keywords: Resistive leakage current, Thermal image, Condition monitoring, Neural network classification. Zinc oxide surge arrester

Copyright (C) 2017 Institute of Advanced Engineering and Science. All rights reserved.

\section{Introduction}

Surge arrester is one of the vital assets in electrical power system. It health condition has a significant impact on the reliable delivery of electricity. However, with substantial growth in demand, high stress in continuous voltages, and many lightning strikes surge arresters have been more severe than before. Meanwhile a large numbers of surge arrester are attaining their designed life or in certain cases operating beyond their design life.

Sometimes electrical company replaced all unit arresters in three phases, although is only one phase of arrester failure. This condition could be high cost it is not always economical to replace all unit with new unit by only considering the condition of one unit arrester. The outcome solution is, continuously monitoring and effective condition assessment is essential for safe and reliable of delivery electricity.

Some researchers have conducted assessment of arrester life monitoring. Wong [1] investigated electromagnetic emission at very high frequency to use as a diagnostic tool of ZnO surge arrester. The chosen of electromagnetic emission is due to high cost in infrared camera, however now a day the infrared or thermography camera is cheap enough and suitable for on line monitoring. Deng Wei et al [2] introduced some improvement of the on-line monitoring of $\mathrm{ZnO}$ surge arrester based on resistive current harmonic. The conclusion stated that the resistive basic harmonic current measuring method is preferable to judge the degradation of the arresters by eliminating the severe electromagnetic interference.

Neto et al [3] developed $\mathrm{ZnO}$ surge arrester monitoring technique my means of continuous analysis of leakage current and thermal image variation. Result shows that thermal image adequate method to monitor $\mathrm{ZnO}$ arrester, however this work is lack of arrester sample and is not enough to prove the technique. Also, Martins, Barbosa et al [4] developed on-site diagnostic technique of $\mathrm{ZnO}$ surge arrester. The technique using thermo vision, leakage current and radio interference were used in on-site measurement. From field measurement results the surge arrester was classify at four categories. The result noticed that every arrester there was abnormalities, however some arrester may be normal or still in good condition but need rigorous inspection. 
Another researcher proposed a monitoring and diagnostic technique using intelligent system. Self-organizing maps which is one kind of artificial neural network used for predictive monitoring $\mathrm{ZnO}$ surge arrester activities studied by [5]. The technique based on utilized of thermal profiles data in SOM artificial neural network. Results shows $95.83 \%$ of testing data was correctly classify. Also Shafi'i and Hamzah proposed infrared thermography data for classify faults of electrical equipment using neural network classification system [6]. The faults classified into four classes as low, intermediate, medium and high and achieved maximum recognition rate $99.38 \%$ in testing phase.

Thermal image for diagnosis faults of $\mathrm{ZnO}$ surge arrester using intelligent system also proposed by [7]. Watershed transformation system was applied to get the segmentation of thermography image surge arrester and neuro-fuzzy network system to classify the condition of surge arrester. Validating system using 100 thermal images and error result was about $10 \%$. Another research in condition monitoring of electrical equipment using artificial neural network and thermal image conducted by [8]. The research used support vector machine (SVM) as machine learning and Zernike moment for extracted thermal image features. The accuracy result was $68.42 \%$.

However, in intelligent condition monitoring system whatever system applied for classification or prediction there is important to consider input features. Features of input play an importance role in the accurate classification process. Good input features give more accurate results compared to the worst input features. Therefore, in the automatic faults diagnosis system, selection of suitable features is very essential to get the accurate information about thermal condition of electrical equipment.

In this research, five features from thermal image were chosen based on the histogram and statistical analysis. The study applied Otsu's statistical threshold selection algorithm for segmented and extracted the features of thermal image. Correlation between thermal image and third harmonic resistive leakage current is a main issue in this research. Three class of condition of surge arrester which are normal, suspicious and fault were classifying using neural network based on the five thermal image features. The supervised neural network is used with third harmonic resistive leakage current as a target of neural network.

\section{Leakage Current Analysis}

The measurement of total leakage current flowing through $\mathrm{ZnO}$ surge arrester under normal conditions is used as one of the health monitoring techniques. However, the total leakage current measurement does not indicate the severity of degradation of $\mathrm{ZnO}$ element as the resistive current (Ir) is only 15-25\% of the total leakage current [9-11]. Hence, a sharp increase in resistive current due to degradation or ageing of $\mathrm{ZnO}$ blocks does not affect the total leakage current considerably. The higher resistive leakage current may ultimately bring in the $\mathrm{ZnO}$ surge arrester to thermal instability and may result in complete failure or breakdown of the arrester. The third harmonic of the resistive leakage current is the true indicator of health of $\mathrm{ZnO}$ surge arrester in service.

Many researchers conducted research to separate the resistive leakage current from the total leakage current [9], [11-13]. The shifted current method claims that it can be separate the resistive leakage current with $95 \%$ accuracy [11]. The method based on waveform manipulation to get capacitive current of leakage current and subtracted it to get the resistive part.

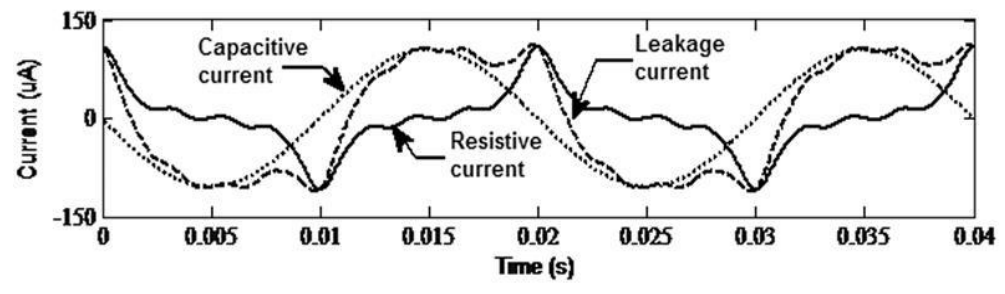

Figure 1. Shifted current method algorithm, dash is total, dotted is capacitive and line is resistive leakage current 
Figure 1 shows the capacitive current was obtained with signal manipulation and resistive current obtained by subtracted total leakage current with capacitive leakage current.

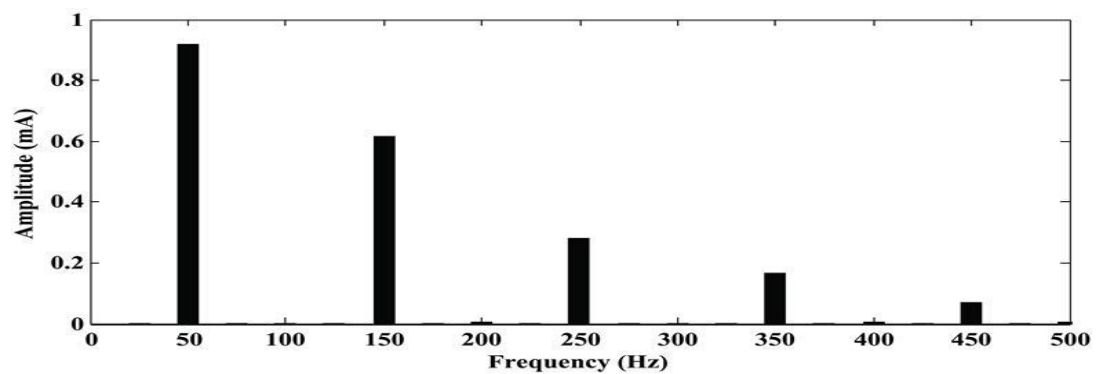

Figure 2. Harmonic spectrum of resistive leakage current, the third harmonic is about $0.61 \mathrm{~mA}$

Figure 2 shows the harmonic spectrum of resistive leakage current obtain using Fast Fourier Transform (FFT), the amplitude of third harmonic is bigger than the others odd harmonic. This parameter could be used as degradation indicator of $\mathrm{ZnO}$ surge arrester.

\section{Thermography image analysis}

Thermography technology is a nondestructive inspection technique [14-17]. The inspection can be conducted efficiently by keeping a distance from the inspected equipment. There is no need to halt equipment operation while an inspection is going on. Since the collection of information for inspection is by telemetry, hazardous operations can be avoided [17]. For these reasons, thermography is widely used for many applications involving preventive maintenance [18]. Thermography technology is based on the detection of infrared radiation emitted by objects with a temperature above absolute zero. Thermography converts this radiation into visible light, resulting in a thermal image. This image is a map of the temperature field on the object's surface which is made possible the power of radiation depends on the radiant property of bodies. Such tests can be performed using thermal cameras.

Nowadays, thermography allows digital recording of the temperature distribution of the test object. This temperature map is interpreted graphically and numeric data. The thermal image of the object is seen on the viewfinder because all temperatures are assigned to a different color. In practice, data is stored as a map of temperatures. The same object may look different depending on the adopted color-scale and relationship to scale of temperatures. Thermal camera system is special kind of thermometer that can measure temperature from a distance, in one moment in many places. It is an effective and noninvasive diagnostic method. Using the thermography cameras obtain temperature field's picture of tested object. The main advantage of this method is that the measurements are made during normal work.

There are many theorems or algorithms to analyze the thermal image. In this the paper, the thermography detection algorithm based on the principle of Otsu's statistical threshold selection algorithm using gray-level histograms [19] is used. The reason for using this algorithm on power facilities is their equipment always has temperatures higher than the environment. By using a thresholding method, the image of $\mathrm{ZnO}$ surge arrester can be separated from its background. Good photographer normally takes picture image that shows a clear distinction between the main object and the background of the picture as shown in Figure 3. The Otsu's statistical threshold selection algorithm can be used to easily separate the object from the background. 


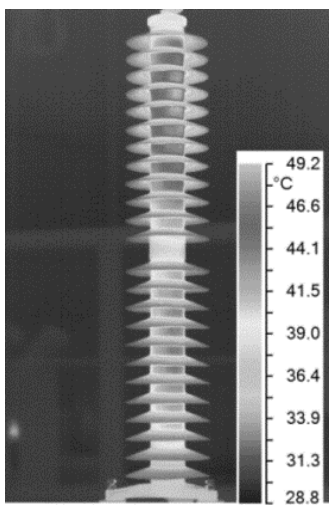

Figure 3. Clear distinction between the main object and the background

Figure 3 shows a clear distinction between the main object and the background of the picture. The picture is gray level $\mathrm{ZnO}$ surge arrester picture taken from high voltage laboratory. Let $\alpha$ represents the original image and $\beta$ represents the extracted binary image of the main object from $\alpha$. As shown in (1), $T$ is the threshold value by Otsu's method;

$W$ is image width and $H$ is image height. Since, this is a digital image, $x$ and $y$ is discrete integers.

$$
\begin{aligned}
& \beta(\mathrm{x}, \mathrm{y})=\left\{\begin{array}{l}
1 \text { if } \alpha(\mathrm{x}, \mathrm{y})>T \\
0 \text { if } \alpha(\mathrm{x}, \mathrm{y}) \leq \mathrm{T}
\end{array}\right. \\
& \forall 0 \leq \mathrm{x}<W, 0 \leq y<H
\end{aligned}
$$

Likewise, all the pixel values of image $\alpha$ are set to 1 when the pixel values are greater than T. On the contrary, the pixel values are set to 0 when they are less than $T$. In order to obtain object image $\mathrm{y}$ from image $\alpha$ and $\beta$, the following formula is used:

$$
\begin{aligned}
& \gamma(\mathrm{x}, \mathrm{y})= \begin{cases}\alpha(x, y) & \text { if } \beta(x, y)=1 \\
0 & \text { if } \beta(x, y)=0\end{cases} \\
& \forall 0 \leq x<W, 0 \leq y<H
\end{aligned}
$$

After the separation process by (1)-(2), $\mathrm{Y}$ is the thermal image of the inspected equipment without background as shown in Figure 8. Excluding the hot area or the brighter spot observed, every part of the main object has a small difference in temperature. After subtracting the hotspots effect, the average temperature of the main object comes close to that of the temperature of the reference point. A morphological image processing theory is adopted in order to extract the hotspots. Firstly, the maximum pixel value of the image $y$ has to be determined as shown in (3).

$$
T(\text { hot }- \text { gray })=\max (\gamma(x, y)) !(0 \leq x W, 0 \leq y<H)
$$

According to the maximum hot value of hot gray, $T$ (hot-gray), an initial seed image $\Omega_{0}$ can be made to calculate the connected components, as shown in (4).

$$
\begin{aligned}
& \Omega_{0}(x, y)=\left\{\begin{array}{c}
1 \quad \text { if } \alpha(x, y)=T_{\text {hot-gray }} \\
0 \quad \text { otherwise }
\end{array}\right. \\
& \forall 0 \leq x<W, 0 \leq y<H
\end{aligned}
$$

Calculate the connected components with the seed image $\Omega 0$ in the foreground image $Y$ with the experiential limit of neighbor's difference of gradient value 16 , as dictated in (5) where $\mathrm{k}$ is the iteration times. $\Omega_{k-1} \oplus B$ expreses a dilation process. If $\Omega_{k-1}(x, y), \forall 0 \leq x<W, 0 \leq y<H$ is equal to 1 then dilated itself according to $\Omega_{k-1}(x+s, y+t), s=t=[-1,1] \mathrm{s}$ and $\mathrm{t}$ are discrete 
integers, due to $B$ is an 8-neighbors mask. $C$ is a constraint of the experiential limit of neighbor's difference of gradient value 16 in the foreground image $y$.

$$
\Omega_{k}=\left(\Omega_{k-1} \oplus B\right) \cap C, k=1,2,3, \ldots
$$

A dilation point would be ineffective if the constraint $C$ not be satisfied. The algorithm has converged when $\Omega k=\Omega k-1$ then gets the converged image $\Omega^{*}$. Finally, $\Omega^{*}$ represents the image of all hotspots' connected components as shown in Figure 9 . Define the every connected component's area of image.

Sometimes, the number of hotspots would be lots because of the characteristic of infrared technology. There are three sources of energy that can be detected from the thermal object; energy emitted from the object itself, energy reflected from the object itself, and energy transmitted by the object [10]. Since infrared imaging technique measures the radiation from the surface of a thermal object, some infrared parameter like emissivity, reflectivity and transmisivity must be accounted for when converting radiation rate into actual temperature values [11]. Some fake hotspots would be generated due to the electrical installation material and special structure that cause the high reflection rate can jeopardize truly infrared emission measurement. Use an Equation (5) to eliminate the noises then search the maximum area of the rests.

\section{Machine learning}

An artificial neural network (ANN) is one kind of machine learning. ANN is simplified models of biological neuron system. It consists of a massively parallel distributed processing system made of highly interconnected neural computing elements called as "Neurons", which has the ability to learn and thereby acquire knowledge. The architecture is inspired from structure of cerebral cortex of brain. ANN comprises of number of neurons which forms the basic processing unit. Each neuron is further connected to other neurons by links. Every neuron receives number of inputs which are modified by 'weights'. The synaptic weights would either strengthen or weaken the signal which is processed further. To generate the final output the sum of the weighted output is passed on to a non-linear filter called as activation function or Transfer function or Squash function, plus a threshold value called bias which releases the output. The output of neural network can be display mathematically as:

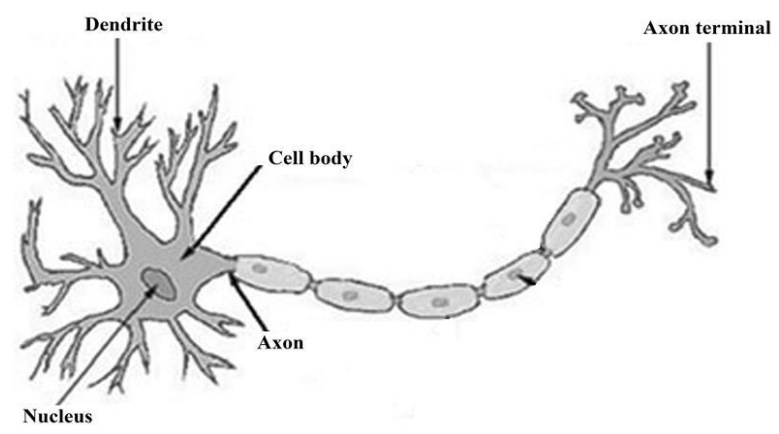

Figure 4. Structure biological neuron, electrical signals from other neurons is conveyed to the cell body by dendrites; resultant electrical signals are sent along the axon to be istributed to other neurons and results in axon terminal.

Figure 4 shows a schematic of oversimplified biological neuron that consist of three major functional units; dendrite, cell body and axon. The cell body has a nucleus that contain information about heredity traits and a plasma that holds the molecular equipment used for producing the material needed by the neuron [20]. The cell body receives signals from the dendrites. The axon terminals, which branches into collaterals receives signal from the cell body and carries them away through the synapse to the dendrites of neighboring neurons. 


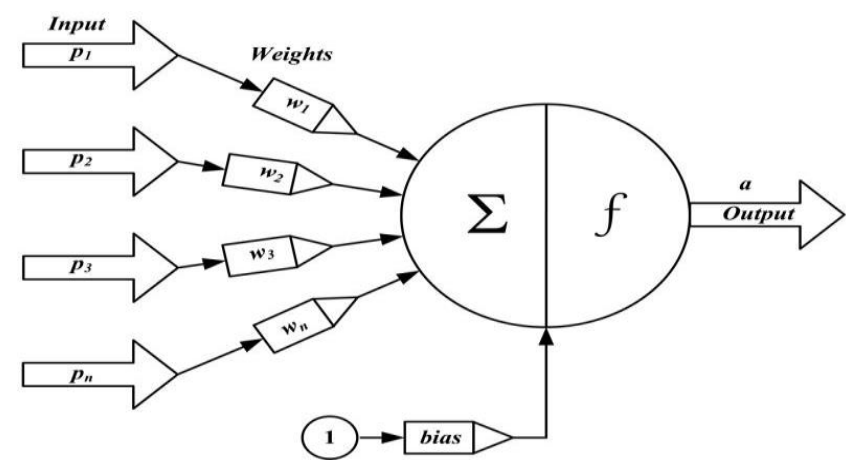

Figure 5. Simple artificial neuron consist of one input layer, weights and bias, function and output layer

Figure 5 shows the connections between nodes which represented the axons and dendrites of analogical artificial neuron equivalent to neuron system. The operation of the artificial neuron is analogous to the operation of the biological neuron: activations from other neurons are summed at the neuron and passed through an activation function, after which the value is sent to other neurons. The input can come from some other units, or perhaps from an external source. Input has an associated weight $\mathrm{w}$, which can be modified so as to model synaptic learning. The unit computes some function $f$ of the weighted sum of its inputs.

$$
a=f\left(\sum p_{i} w_{i}+b\right)
$$

Neural networks are made up of many artificial neurons. An artificial neuron is simply an electronically modeled biological neuron. How many neurons are used depends on the task at hand. It could be as few as three or as many as several thousand. One optimistic researcher has even hard wired 2 million neurons together in the hope he can come up with something as intelligent as a cat although most people in the artificial intelligent community. There are many different ways of connecting artificial neurons together to create a neural network but I shall be concentrating on the most common which is called a feedforward network.

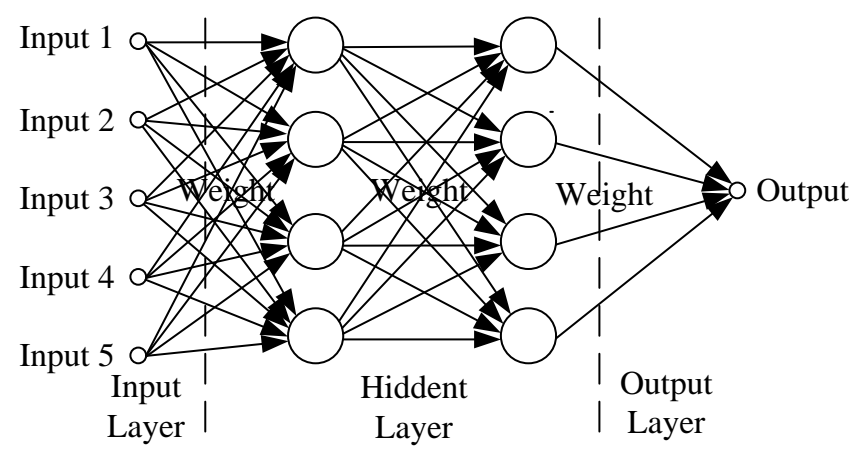

Figure 6. Feedforward multylayer perceptron neural network architecture

There are many types of ANN architectures; however they consist of two big categories as feedforward neural network and recurrent or feedback neural network. Feedforward neural network are single layer perceptron (SLP), multilayer perceptron (MLP) and radial basis function (RBF) neural network and recurrent or feedback neural network are competitive network, kohonen's SOM, Hopfield network and ART model. MLP is the most popular network due to its computional simplicity, finite parameterization, stability and small size for a particular problem compared to other architectures [15]. 


\section{Research method}

The research method is explaining research chronological, including research design, research procedure.

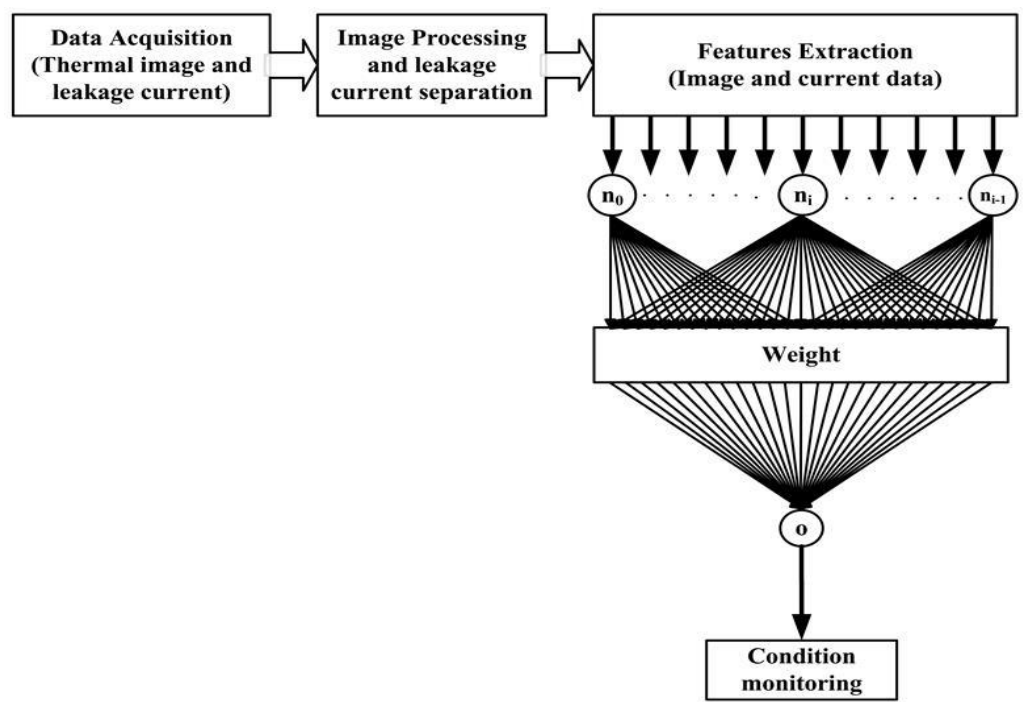

Figure 7. Methodology of $\mathrm{ZnO}$ arrester condition monitoring. Leakage current as well as thermal image were used for training input. The condition of arrester was used as an output

(normal, caution and faulty)

Figure 7 shows the methodology was used in this research. Thermal image data and leakage current were captured in same time. The thermal images and leakage current signals were stored as data acquisition. Each data was processed separately and extracted some features for neural network input and target.

The leakage currents were processed using shifted current method and fast fourier transform (FTT) to obtain the third harmonic resistive leakage current and used as a target of neural network. And the thermal images were processed using Otsu's statistical threshold selection method to get some features eg.: maximum, minimum, mean, standard deviation and variance and used input of neural network.

The architecture of neural network in this work is a back propagation neural network with resilient back propagation training function (trainrp). The resilient back propagation is a network training function that updates weight and bias values according to the resilient back propagation algorithm. The goal of neural network is classify the condition of $\mathrm{ZnO}$ surge arrester as normal, caution and faulty.

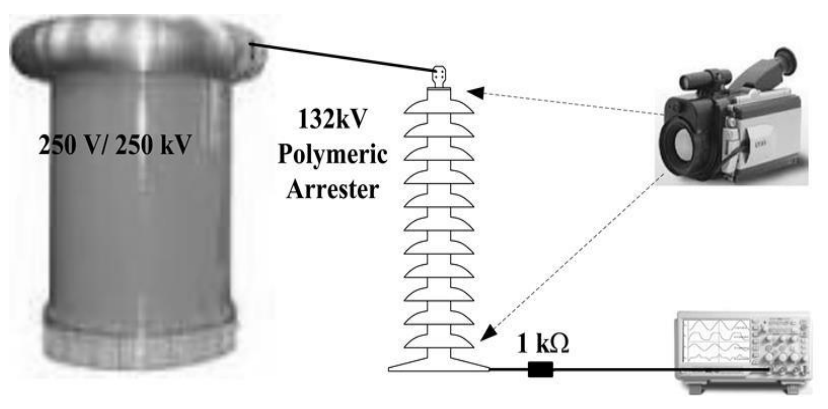

Figure 8. Experimental setup for energisation and thermal imaging of 132kV-rated $\mathrm{ZnO}$ arresters $1 \mathrm{kohm}$ shunt resistor is used for leakage current. 
Figure 8 shows a $132 \mathrm{kV}$ polymeric $\mathrm{ZnO}$ surge arrester energized by the $250 \mathrm{kV}$ phaseto-ground high voltage test system from $70 \mathrm{kV}$ to $120 \mathrm{kV}$ with step $1 \mathrm{kV}$, this technique to see possible deterioration in service, the peak value of the total leakage current that flows through the surge arrester when it is subjected to the test voltage, is measured. The test was performed by applying a voltage that was raised, from a value sufficiently low up to reach the test voltage. The peak value of the leakage current should be measured through the voltage drop on a resistive shunt $(1 \mathrm{k} \Omega)$ connected in series with the surge arrester. The voltage drop on shunt resistor was measured by an oscilloscope and current waveforms were recorded for further analysis. The measurement set up for thermography image and leakage current can be seen in Figure 7.

\section{Results and analysis}

Experiment was done in UTM High Voltage and High Current (IVAT) Laboratory. Station $\mathrm{ZnO}$ arrester was apply continuous voltage from 80 to $110 \mathrm{kV}$. For every $1 \mathrm{kV}$, some data such rms and peak and waveform were recorded and save to further analysis. Figure 8 shows the waveform of total leakage current $\mathrm{ZnO}$ surge arrester when applied voltage was $105 \mathrm{kV}$.

\subsection{Signal Analysis and Feature Extracting}

A Sample of total leakage current which is obtained from laboratory experiment is shown on Figure 9. The waveform of total leakage current $\mathrm{ZnO}$ surge arrester is captured when applied voltage was 105kV. It was done in Universiti Teknologi Malaysia High Voltage and High Current (IVAT) Laboratory. Station ZnO arrester was apply continuous voltage from 70 to 120 $\mathrm{kV}$. For every $1 \mathrm{kV}$, some data such rms and peak and waveform were recorded as shown in Table 1 and save to further analysis. Figure 9 shows

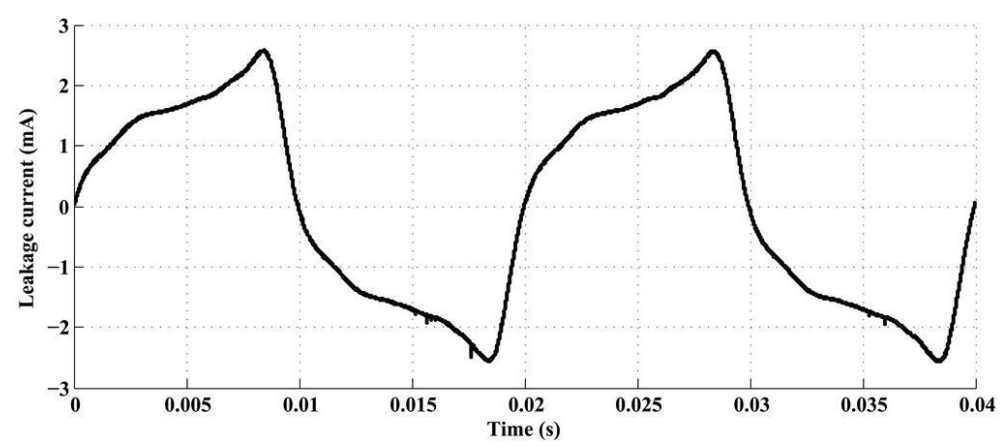

Figure 9. A sample of total leakage current oscillogram (applied voltage is $105 \mathrm{kV} \mathrm{rms).}$

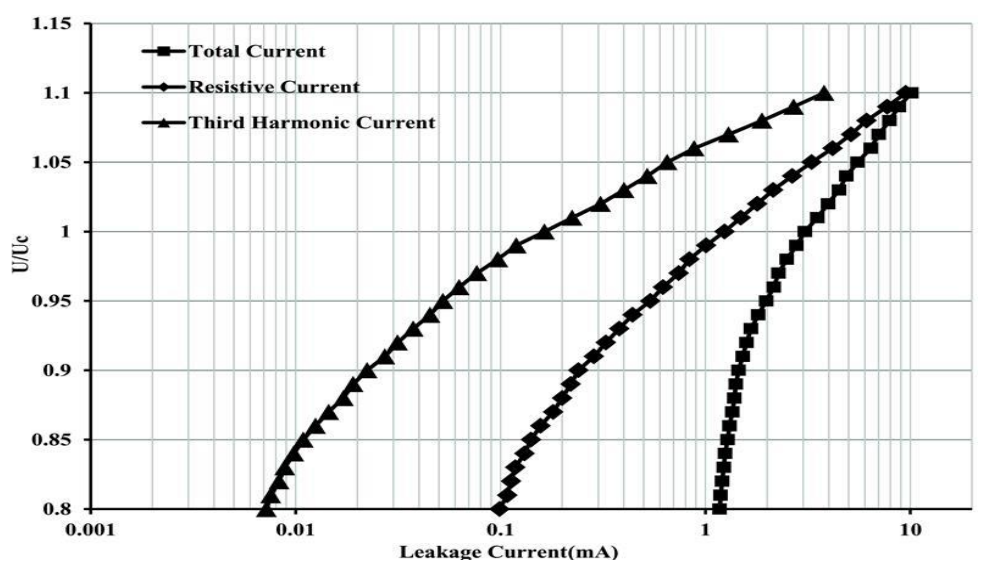

Figure 10. Logarithmic plot of total, resistive and third harmonic leakage current 
Measurement and processed data are shown in Table 1. Total leakage current was taken from laboratory measurement, the resistive leakage current was obtained using shifted current method and the third harmonic resistive leakage current was obtained using FFT of resistive current. The logarithmic plotting of all parameter can be show in Figure 9.

Table 1. Results of total, resistive and third harmonic of leakage current zno surge arrester for sample 1

\begin{tabular}{|c|c|c|c|c|}
\hline $\begin{array}{c}\text { Voltage } \\
(\mathrm{kV})\end{array}$ & $\begin{array}{c}\text { Total LC } \\
(\mathrm{mA})\end{array}$ & $\begin{array}{l}\text { Resistive } \\
\text { LC (mA) }\end{array}$ & $\begin{array}{c}\text { Third } \\
\text { Harmonic } \\
\text { LC }(m A)\end{array}$ & $\begin{array}{c}\text { Ambient } \\
\text { Temp. (oC) }\end{array}$ \\
\hline 70 & 0.795 & 0.2024 & 0.0526 & 27.10 \\
\hline 71 & 0.815 & 0.2072 & 0.0554 & 27.20 \\
\hline 72 & 0.829 & 0.2091 & 0.0584 & 27.30 \\
\hline 73 & 0.832 & 0.2170 & 0.0618 & 27.40 \\
\hline 74 & 0.842 & 0.2241 & 0.0649 & 27.70 \\
\hline 75 & 0.854 & 0.2342 & 0.0693 & 27.90 \\
\hline 76 & 0.869 & 0.2433 & 0.0721 & 28.10 \\
\hline 77 & 0.887 & 0.2537 & 0.0752 & 28.20 \\
\hline 78 & 0.891 & 0.2624 & 0.0772 & 28.30 \\
\hline 79 & 0.907 & 0.2729 & 0.0815 & 28.40 \\
\hline 80 & 0.918 & 0.2829 & 0.0856 & 28.50 \\
\hline 81 & 0.939 & 0.2927 & 0.0873 & 28.70 \\
\hline 82 & 0.944 & 0.3014 & 0.0897 & 28.70 \\
\hline 83 & 0.956 & 0.3132 & 0.0949 & 28.80 \\
\hline 84 & 0.974 & 0.3255 & 0.0988 & 28.90 \\
\hline 85 & 0.983 & 0.3357 & 0.1062 & 29.10 \\
\hline 86 & 0.998 & 0.3497 & 0.1082 & 29.30 \\
\hline 87 & 1.019 & 0.3515 & 0.1132 & 29.40 \\
\hline 88 & 1.020 & 0.3629 & 0.1147 & 29.50 \\
\hline 89 & 1.031 & 0.3729 & 0.1208 & 29.60 \\
\hline 90 & 1.061 & 0.3815 & 0.1233 & 29.70 \\
\hline 91 & 1.066 & 0.3915 & 0.1293 & 29.70 \\
\hline 92 & 1.074 & 0.4045 & 0.1396 & 29.80 \\
\hline 93 & 1.094 & 0.4151 & 0.1495 & 29.80 \\
\hline 94 & 1.124 & 0.4251 & 0.1562 & 29.90 \\
\hline 95 & 1.131 & 0.4361 & 0.1641 & 30.00 \\
\hline 96 & 1.137 & 0.4462 & 0.1721 & 30.10 \\
\hline 97 & 1.154 & 0.4696 & 0.1794 & 30.20 \\
\hline 98 & 1.161 & 0.4959 & 0.1902 & 30.30 \\
\hline 99 & 1.188 & 0.5180 & 0.1964 & 30.40 \\
\hline 100 & 1.209 & 0.5390 & 0.2114 & 30.60 \\
\hline 101 & 1.225 & 0.5596 & 0.2251 & 30.70 \\
\hline 102 & 1.248 & 0.5814 & 0.2359 & 30.80 \\
\hline 103 & 1.272 & 0.6119 & 0.2442 & 30.90 \\
\hline 104 & 1.280 & 0.6333 & 0.2598 & 31.00 \\
\hline 105 & 1.329 & 0.6790 & 0.2729 & 31.00 \\
\hline 106 & 1.354 & 0.7270 & 0.2973 & 31.10 \\
\hline 107 & 1.374 & 0.7765 & 0.3192 & 31.10 \\
\hline 108 & 1.408 & 0.8169 & 0.3493 & 31.20 \\
\hline 109 & 1.483 & 0.8987 & 0.3688 & 31.20 \\
\hline 110 & 1.541 & 0.9867 & 0.3914 & 31.30 \\
\hline 111 & 1.642 & 1.1151 & 0.4348 & 31.40 \\
\hline 112 & 1.790 & 1.2038 & 0.4718 & 31.50 \\
\hline 113 & 1.945 & 1.3322 & 0.5286 & 31.50 \\
\hline 114 & 2.118 & 1.5117 & 0.5818 & 31.60 \\
\hline 115 & 2.298 & 1.6195 & 0.6360 & 31.50 \\
\hline 116 & 2.521 & 1.8080 & 0.7067 & 31.50 \\
\hline 117 & 2.726 & 2.0136 & 0.7665 & 31.40 \\
\hline 118 & 2.987 & 2.4336 & 0.8324 & 31.30 \\
\hline 119 & 3.478 & 2.8499 & 0.9707 & 31.20 \\
\hline 120 & 4.220 & 3.9044 & 1.0754 & 31.10 \\
\hline
\end{tabular}

Figure 10 show the plotting of Table 1 in logarithmic scale, with y axis is per unit voltage and $x$ axis is value of the leakage current in milliampere. It shows the third harmonic resistive leakage current (triangle mark), the resistive leakage current (diamond mark) and total leakage current (cube mark) at the knee point have same value. 


\subsection{Image Processing and Features Extracting}

The Otsu's segmentation is shown in Figure 11. Figure 10(a) is $\mathrm{ZnO}$ thermal image result when applied with voltage $110 \mathrm{kV}$. Figure $11(\mathrm{~b})$ is gray level image resulted from Otsu's processed and Figure 10(c) is segmented results of thermal image using Otsu's method.

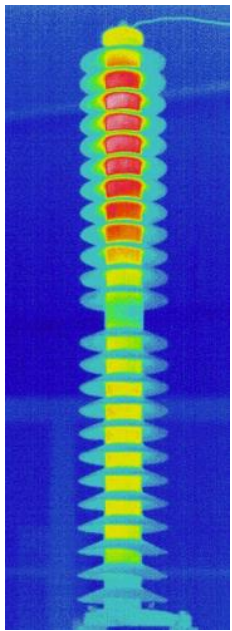

(a)

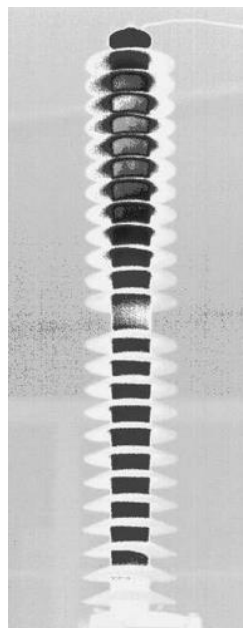

(b)

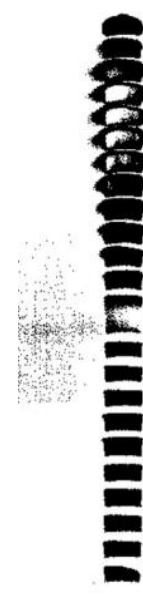

(c)

Figure 11. (a) Thermal image $\mathrm{ZnO}$ arrester when applied voltage $110 \mathrm{kV}$, (b) Grayscale thermal image and (c) Thermal image segmentation using Otsu's statistical threshold selection algorithm

Image segmented thermal histogram is shown in Figure 12. From this histogram some features were extracted such as maximum, minimum, mean and standard deviation as shown in Table 2.

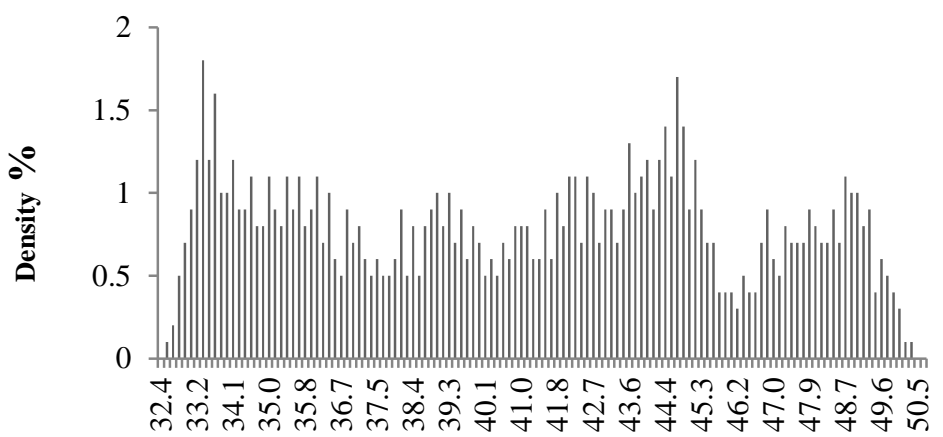

Temperature $\left({ }^{\circ} \mathrm{C}\right)$

Figure 12. Histogram of image segmentation using Otsu's statistical threshold selection algorithm

Table 2. Features extracted from thermal image

\begin{tabular}{lcccccc}
\hline Parameter & 1 & 2 & 3 & 4 & $\ldots$ & $\mathrm{n}$ \\
\hline Maximum & 40.179 & 38.667 & 37.676 & 37.3 & $\ldots$ & 41.344 \\
Minimum & 32.831 & 31.506 & 30.541 & 30.054 & $\ldots$ & 30.942 \\
$\quad$ Mean & 36.477 & 35.059 & 34.081 & 33.677 & $\ldots$ & 36.143 \\
Standard & 4.5423 & 4.3098 & 4.279 & 4.4794 & $\ldots$ & 9.2327 \\
deviation & & & & & &
\end{tabular}




\subsection{Training And Testing Neural Network}

Figure 13 shows training performance at epoch 241. It can be seen that goal performance at 0.0001 is archived. Although has some fluctuations but in general this good performance of neural network training.

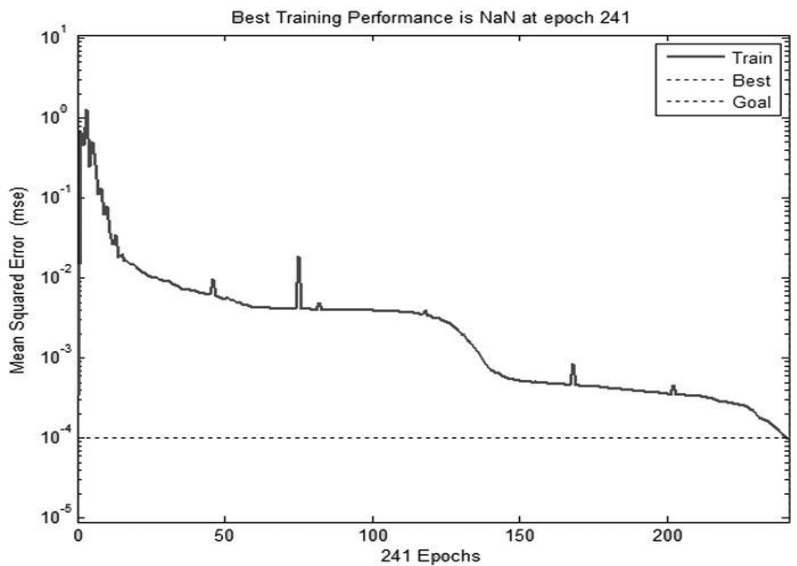

Figure 13. Evolution of the MSE in 241 epochs. Dot is goal setting at 0.0001 , line is train performance

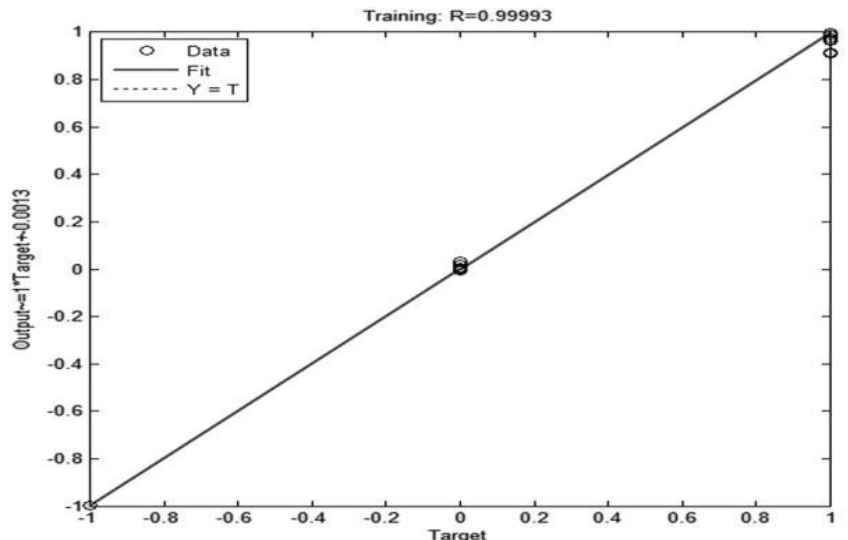

Figure 14. Linear regression analysis of neural network training regression is 0.99993

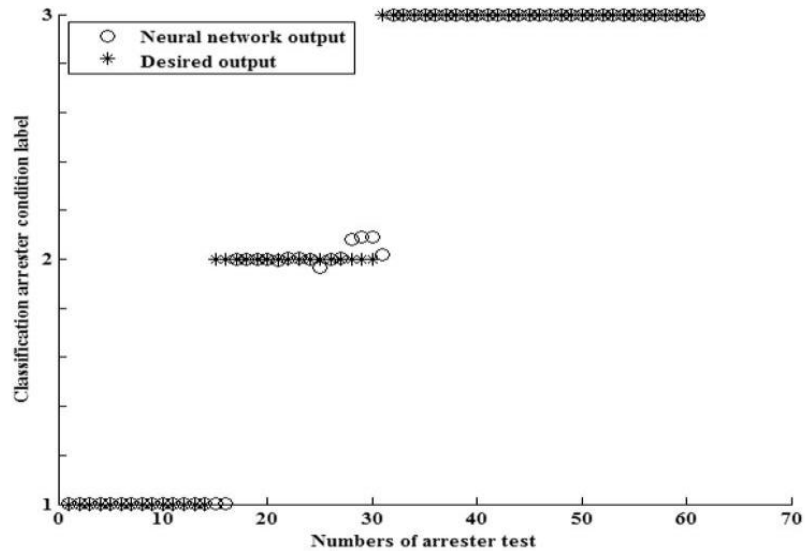

Figure 15. ANN testing results of arrester classification ( 1 normal, 2 suspicious, and 3 faulty) based on thermal imaging against the known target (desired output) 
Figure 15 shows of 61 set thermal image data testing. Neural network testing results shows of 3 wrong classifications that are image sample 16 and 17 classified as 1 instead of 2 , and image sample 31 classified as 2 instead of 3 . The rest of image samples were classified as desire output.

\section{Conclusion}

The third harmonic resistive leakage current as indicator of $\mathrm{ZnO}$ surge arrester aging has been successful extracted from total leakage current using shifted current method. Some features of thermal image such as maximum, minimum, mean and standard deviation have obtained by applied histogram of Otsu's segmented grayscale thermal image method. Neural network training archives goal performance at 241 epochs with training regression is 0.99 . And testing with 61 set thermal image data recorded small error which is only 3 set data is not classify correctly.

\section{Acknowledgements}

The authors would like to thank Engineering Faculty Andalas University Padang Indonesia for the financial and management support provided under Contract No. 010/UN.16.09.D/PL/2017.

\section{References}

[1] KL Wong, Y Xiang, S Nahavandi. Emission-based condition monitoring technique for ZnO surge arrester. Series on Energy and Power Systems. pp. 234-237.

[2] W Deng, W Liu, Z Fu et al. The Network-model On-line Monitoring System of ZnO Surge Arresters in 500kV Substation. pp. 1-5.

[3] ETW Neto, EG Da Costa, R Trajano de Souza et al. Monitoring and Diagnosis of ZnO Arresters. Latin America Transactions, IEEE (Revista IEEE America Latina), vol. 4, no. 3, pp. 170-176, 2006.

[4] H Martins, FB Barbosa, W Cruz, Evaluation and diagnosis technique for surge arresters. pp. 614617.

[5] G. R. S. Lira, E. G. Costa, C. W. D. Almeida. Self-organizing maps applied to monitoring and diagnosis of $\mathrm{ZnO}$ surge arresters. pp. 659-664.

[6] M. A. Shafi'i, and N. Hamzah. Internal fault classification using Artificial Neural Network. pp. 352-357.

[7] C. A. Laurentys Almeida, A. P. Braga, S. Nascimento et al., Intelligent Thermographic Diagnostic Applied to Surge Arresters: A New Approach. Power Delivery, IEEE Transactions on, vol. 24, no. 2, pp. 751-757, 2009.

[8] A. Rahmani, J. Haddadnia, O. Seryasat, Intelligent fault detection of electrical equipment in ground substations using thermo vision technique. pp. V2-150-V2-154.

[9] J. Lundquist, L. Stenstrom, A. Schei et al., New method for measurement of the resistive leakage currents of metal-oxide surge arresters in service, Power Delivery, IEEE Transactions on, vol. 5, no. 4, pp. 1811-1822, 1990.

[10] D. A. Silva, E. C. M. Costa, J. L. Franco et al., Polymer surge arresters: Degradation versus electrical performance. pp. 63-68.

[11] Z. Abdul-Malek, Novizon, Aulia, A new method to extract the resistive component of the metal oxide surge arrester leakage current. pp. 399-402.

[12] B.-H. Lee, S.-M. Kang. A new on-line leakage current monitoring system of $\mathrm{ZnO}$ surge arresters. Materials Science and Engineering B, vol. 119, no. 1, pp. 13-18, 2005.

[13] A. Gakiya Kanashiro, M. Zanotti, P. Futoshi Obase et al. Diagnostic of silicon carbide surge arresters using leakage current measurements, Latin America Transactions, IEEE (Revista IEEE America Latina), vol. 9, no. 5, pp. 761-766, 2011.

[14] A. Bhatt, D. Pant, R. Singh. An analysis of the performance of Artificial Neural Network technique for apple classification. AI \& SOCIETY, pp. 1-9, 2012/05/01, 2012.

[15] K. Mahmood, A. Zidouri, A. Zerguine. Performance analysis of a RLS-based MLP-DFE in timeinvariant and time-varying channels. Digital Signal Processing, vol. 18, no. 3, pp. 307-320, 5//, 2008.

[16] A. Kavithamani, V. Manikandan, N. Devarajan. Soft Fault Classification of Analog Circuits Using Network Parameters and Neural Networks. Journal of Electronic Testing, vol. 29, no. 2, pp. 237-240, 2013/04/01, 2013.

[17] D. Li, B. Du, L. Zhang. The Design of the Weeds Classification System Based on BP Neural Network. Advances in Computer Science and Information Engineering, Advances in Intelligent and Soft Computing. D. Jin and S. Lin, eds., pp. 625-630: Springer Berlin Heidelberg, 2012. 
[18] D. Chen, C. Zhang, Z. Zeng et al. Active infrared thermal imaging technology to detect the corrosion defects in aircraft cargo door. pp. 751206-9.

[19] M. G. Augasta, T. Kathirvalavakumar. A Novel Pruning Algorithm for Optimizing Feedforward Neural Network of Classification Problems. Neural Processing Letters, vol. 34, no. 3, pp. 241-258, 2011/12/01, 2011.

[20] S. Becker, M. Plumbley. Unsupervised neural network learning procedures for feature extraction and classification. Applied Intelligence, vol. 6, no. 3, pp. 185-203, 1996/07/01, 1996. 